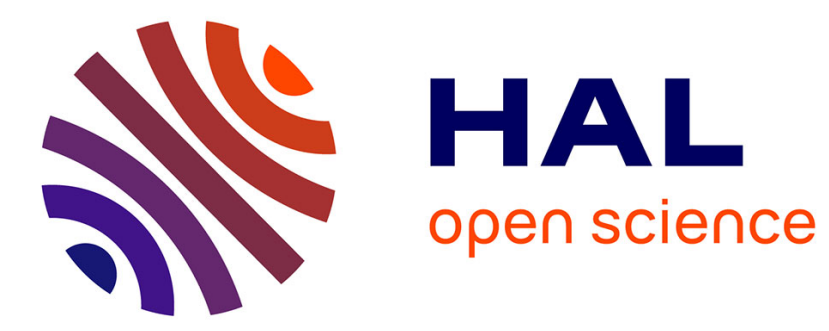

\title{
Experiments and simulations of a gravitational granular flow instability
}

\author{
Jan Ludvig Vinningland, Øistein Johnsen, Eirik G. Flekkøy, Renaud \\ Toussaint, Knut Jørgen Måløy
}

\section{- To cite this version:}

Jan Ludvig Vinningland, Øistein Johnsen, Eirik G. Flekkøy, Renaud Toussaint, Knut Jørgen Måløy. Experiments and simulations of a gravitational granular flow instability. Physical Review E : Statistical, Nonlinear, and Soft Matter Physics, 2007, 76 (5), 10.1103/PhysRevE.76.051306 . hal-01909770

\section{HAL Id: hal-01909770 \\ https://hal.science/hal-01909770}

Submitted on 31 Oct 2018

HAL is a multi-disciplinary open access archive for the deposit and dissemination of scientific research documents, whether they are published or not. The documents may come from teaching and research institutions in France or abroad, or from public or private research centers.
L'archive ouverte pluridisciplinaire HAL, est destinée au dépôt et à la diffusion de documents scientifiques de niveau recherche, publiés ou non, émanant des établissements d'enseignement et de recherche français ou étrangers, des laboratoires publics ou privés. 


\title{
Experiments and simulations of a gravitational granular flow instability
}

\author{
Jan Ludvig Vinningland, ${ }^{1, *}$ Øistein Johnsen, ${ }^{1}$ Eirik G. Flekkøy, ${ }^{1}$ Renaud Toussaint, ${ }^{2}$ and Knut Jørgen Måløy ${ }^{1}$ \\ ${ }^{1}$ Department of Physics, University of Oslo, P.0. Box 1048, 0316 Oslo, Norway \\ ${ }^{2}$ Institut de Physique du Globe de Strasbourg, CNRS, Université Louis Pasteur, 5 rue Descartes, 67084 Strasbourg Cedex, France
}

(Received 1 November 2006; revised manuscript received 28 September 2007; published 27 November 2007)

\begin{abstract}
An instability is observed as a layer of dense granular material positioned above a layer of air falls in a gravitational field [Phys. Rev. Lett. 99, 048001 (2007)]. A characteristic pattern of fingers emerges along the interface defined by the grains, and a transient coarsening of the structure is caused by a coalescence of neighboring fingers. The coarsening is limited by the production of new fingers as the separation of the existing fingers reaches a certain distance. The experiments and simulations presented are shown to be comparable both qualitatively and quantitatively. The characteristic inverse length scale of the structures, obtained as the mean of the solid fraction power spectrum, relaxes toward a stable value shared by the numerical and experimental data. Further, the response of the numerical model to changes in various model parameters is investigated. These parameters include the density of the grains, the shape of the initial air-grain interface, and the dissipation of the granular phase. Also, the growth rates of the bulk solid fraction and the air-grain interface are obtained from Fourier power spectra of the numerical data. This analysis reveals that the instability is never in a linear regime, not even initially.
\end{abstract}

DOI: 10.1103/PhysRevE.76.051306

\section{INTRODUCTION}

Sands and powders are indispensable materials in our modern society. Nevertheless, a complete theoretical description of granular materials and their dynamical properties are still not available, despite their widespread use. An improved understanding of granular flows would provide valuable contributions to many industrial applications such as pneumatic transport, fluidized beds, and catalytic cracking [1-3]. Granular materials are also involved in a host of natural and geological phenomena, most of which are still in need of a proper understanding. Such phenomena include sedimentation [4,5], erosion and river evolution [6], underwater avalanches and turbidities [7], and soil fluidization during earthquakes [8].

The relatively complete description of classical fluid dynamics is also useful in the interpretation of granular flows. Instability is a central problem in fluid dynamics $[9,10]$, and over the past few years some classical hydrodynamic instabilities have also been reported in granular systems. Examples of such are the Saffman-Taylor instability [11] in a granular suspension [12] or in gas-grain mixtures [13], the Kelvin-Helmholtz instability [14] in a vibrated granular mixture [15], the Richtmyer-Meshkov instability in a shock propagating at the interface between two types of grains [16], as well as some novel instabilities in submarine avalanches [17] and in a tube of sand $[18,19]$. The granular RayleighTaylor instability discussed here was first reported by the same authors in Ref. [20]. The present paper provides a more elaborate and complete discussion of the instability in addition to further results and analyses.

While we study a gas-grain system, the liquid-grain interface in a Hele-Shaw cell was investigated experimentally and theoretically by Völtz et al. [21]. Sieved glass beads of $80 \mu \mathrm{m}$ in diameter was used in their experiments, and a be-

\footnotetext{
*janlv@fys.uio.no
}

PACS number(s): 81.05.Rm, 47.20.Ma, 47.11.-j, 45.70.Qj

havior very similar to the classical Rayleigh-Taylor instability $[10,22]$ was observed. In contrast, the instability discussed in this paper arises in a liquid-free granular material and displays processes of tip splitting and finger renucleation. Our experimental and numerical data compare favorably both qualitatively and quantitatively, despite the twodimensionality of the model and the neglect of interparticle friction and gas inertia. Linear stability analysis is not adequate to predict the final dominating wavelength because nonlinear effects govern the selection of the final wavelength. This is demonstrated explicitly in the numerical data as the growth rates of the solid fraction wave numbers are shown not to follow an exponential growth of any significant duration. The parameter space of the model is explored by changing the density of the grains, the shape of the initial granular interface, and the granular dissipation.

The motivation for the simulations and experiments presented in this paper is to study a granular version of the Rayleigh-Taylor instability [22] known from hydrodynamics, where a dense Newtonian liquid is positioned on top of a less dense liquid in a gravitational field. In our case the dense fluid is replaced by a granular material which, in contrast to the liquid, is not subject to surface tension.

The simulation of two-phase flow is particularly interesting for many engineering purposes, and historically most simulations of two-phase flows have treated the solid phase as a continuum, which allowed numerical techniques known from fluid dynamics to be applied. A continuum approach to granular media (see, e.g., Ref. [23]) is, however, only approximate, and will in cases of discontinuous density variations break down completely. Moreover, many interesting phenomena in granular media are closely related to its particularity. A complete description of the interactions between a continuum fluid phase and a discrete solid phase would require the full Navier-Stokes equation for the fluid, coupled with moving boundary conditions given by the surfaces of the grains and the geometry of the container, together with differential equations for the grains. Such a detailed scheme would consume prohibitive computational resources, and a 


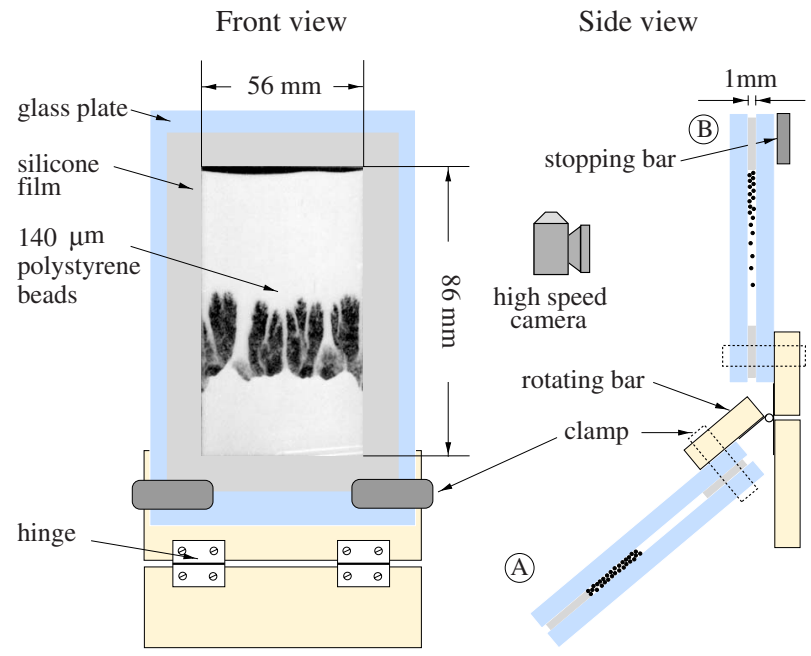

FIG. 1. (Color online) Illustration of the experimental setup viewed from the front and the side. Two cell positions are superimposed in the side view to demonstrate the manual rotation from position $A$ to $B$.

number of techniques (see Ref. [24] for a brief summary) have been developed in order to reduce the computational efforts while preserving the physics. In contrast, we use a hybrid technique that affords a continuum description of the gas phase and a particulate description of the granular phase. Our model neglects friction, the spatial direction normal to the cell, and the inertia of the air. Yet, this computationally agile model yields results in good agreement with experimental observations.

The paper is organized as follows. Section II presents the setup and execution of the experiment, followed by an outline of the numerical model and its implementation in Sec. III. The numerical and experimental results are presented and analyzed in Sec. IV, which is split into four subsections devoted to qualitative descriptions of the instability (Sec. IV A), numerical solid fraction and pressure profiles (Sec. IV B), Fourier power spectra and growth rate analysis (Sec. IV C), and the variation of the model parameters (Sec. IV D). The paper is completed with a short conclusion in Sec. V.

\section{EXPERIMENT}

The experimental setup, illustrated in Fig. 1, consists of a closed Hele-Shaw cell mounted on a hinged bar which enables the cell to rotate $130^{\circ}$ from a lower to an upper vertical position (from $A$ to $B$ in Fig. 1). The Hele-Shaw cell is made of two 8-mm-thick glass plates clamped onto a 1-mm-thick silicone frame. The internal dimensions of the cell are 56 $\times 86 \times 1 \mathrm{~mm}^{3}$, and it is filled with polystyrene beads and air at atmospheric pressure. The cell is rotated manually and it takes about $0.2 \mathrm{~s}$ to bring the cell to an upright, vertical position. The off-center pivot of the cell cause the rotation to slow down the falling motion of the grains due to centrifugal forces. A bar is mounted at position $B$ in order to control the final vertical position of the cell. However, this bar has some undesirable side effects, causing nonpersistent perturbations of the initial stages of the instability (see Sec. IV A). The full development of the instability is recorded by a high-speed digital camera (Photron Fastcam-APX 120K) taking images with a resolution of $512 \times 512$ pixels at a rate of 500 frames per second.

Monodisperse polystyrene beads of $140 \mu \mathrm{m}$ in diameter from Microbeads ${ }^{\circledR}$ (Dynoseeds TS 140-51) are used in the experiments. The filling of the cell is performed with one glass plate lying down horizontally and the silicone frame adhered on top. Small portions of beads are carefully deposited on the plate and leveled with the silicone frame before the upper plate is attached and fastened with clamps. The cell is flipped a few times after closure to allow the grains to form a random loose-packed configuration.

The humidity in the laboratory is important in order to keep the electrostatic and cohesive properties of the grains at a suitable level to prevent the grains from clustering or sticking to the glass plates. During the filling of the cell and the execution of the experiment the humidity was kept constant at about $30 \%$.

The experimental results are presented and discussed together with the numerical results in Sec. IV.

\section{SIMULATION}

\section{A. Model}

The numerical model is a hybrid model that combines a discrete description of the grains with a continuum description of the gas. The foundation and derivation of the model are presented in detail in Refs. [25,26]; only an outline of the model is given here.

The granular phase is considered as an agglomeration of spheres that makes up a deformable porous medium which in our model is described by coarse-grained solid fraction $\rho(x, y)$ and velocity fields $\mathbf{u}(x, y)$, where $(x, y)$ are the twodimensional space coordinates. The continuum gas phase is solely described by its pressure field $P(x, y)$; hence the inertia and velocity field of the gas are not considered in this model. The focus of the model is to describe the granular flow on a scale of a few grain diameters, not the fluid flow field on a subgranular level. It is justified to neglect the inertia of the fluid as long as the Reynolds number is small, which is the case for particles on a micrometer scale sedimenting in air.

The pressure is governed by the equation $[25,26]$

$$
\phi\left(\frac{\partial P}{\partial t}+\mathbf{u} \cdot \nabla P\right)=\nabla \cdot\left(P \frac{\kappa}{\mu} \nabla P\right)-P \nabla \cdot \mathbf{u},
$$

where $\phi=1-\rho$ is the porosity, $\rho$ is the solid fraction, $\kappa$ is the permeability, and $\mathbf{u}$ is the velocity field of the granular phase. $P$ is the gas pressure, and $\mu$ is the gas viscosity. This equation is derived from the conservation of air and grain mass, using Darcy's law [27] to obtain the pressure drop over a given volume described by a permeability $\kappa$. The CarmanKozeny relation [28] is assumed for the permeability, and the isothermal ideal gas law is assumed for the compressible gas. The empirical Carman-Kozeny relation is given as 


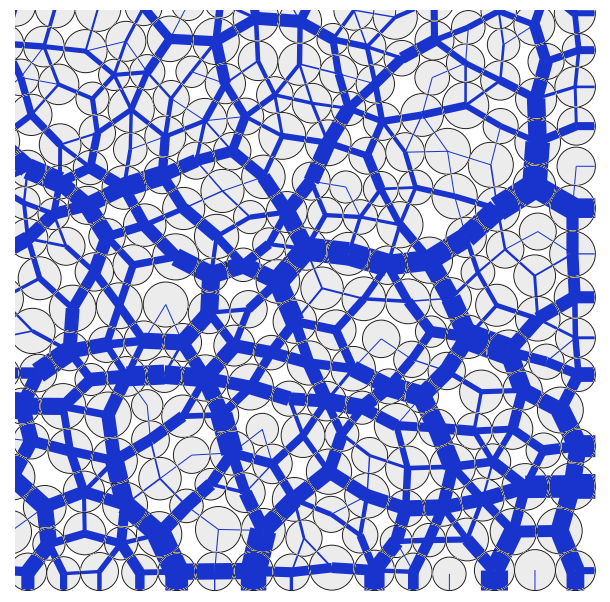

FIG. 2. (Color online) Network of interparticle forces $\mathbf{F}_{\mathrm{I}}$ calculated by contact dynamics. This image shows the lower right corner of a frictionless packing of 1200 grains relaxed under gravity.

$$
\kappa(\rho, d)=\frac{d^{2}}{180} \frac{(1-\rho)^{3}}{\rho^{2}},
$$

where $\rho$ is the local solid fraction, $d$ is the diameter of the grains, and 1/180 is an empirical constant valid for a packing of beads.

The dynamics of the grains are governed by Newton's second law

$$
m \frac{d \mathbf{v}}{d t}=m \mathbf{g}+\mathbf{F}_{\mathrm{I}}-\frac{\nabla P}{\rho_{n}},
$$

where $m$ is the grain mass, $\mathbf{v}$ is the grain velocity, $\mathbf{F}_{\mathrm{I}}$ is the interparticle force which keeps the grains from overlapping, $\rho_{n}=\rho \rho_{g} / m$ is the number density, and $\rho_{g}$ is the mass density of the material the grains are made of. Contact dynamics [29] is used to calculate the interparticle force $\mathbf{F}_{\mathrm{I}}$ but molecular dynamics techniques [30,31] could have been used instead. Contact dynamics is an iterative scheme that calculates the interparticle distribution of normal forces consistent with the kinematic constraints imposed by the impenetrable beads. The solution yields a force network that satisfy volume exclusion at every contact. The relative force change from one iterative step to the next is used as a convergence criterion: If the change at every contact is less than a given threshold the solution has converged. The resulting force network shows how the weight of the grains, and possible external forces such as the gas pressure, are transmitted and carried by the grains and the container. More detailed descriptions of contact dynamics are found in Refs. [32-34]. Figure 2 gives an example a force network obtained in a frictionless packing of 1200 grains relaxed under gravity. The direction and magnitude of the forces are respectively given by the direction and the thickness of the lines. Typical features of a force network are the heterogeneous distribution of forces and the existence of force chains that transmit a major fraction of the load.

The interparticle and wall-particle friction are not taken into account in this model. The dissipation of the granular phase is controlled by a coefficient of normal restitution, $\varepsilon$,

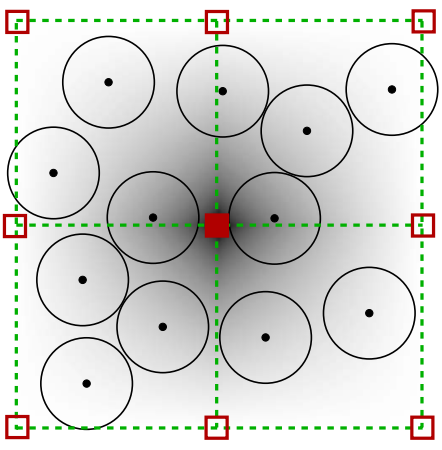

FIG. 3. (Color online) Illustration of the linear smoothing function used to calculate the continuous fields from the discrete positions and velocities of the grains. The red squares are nodes on the grid, and the gray-level background illustrates the weight map of the central node; black is 1 , white is zero.

that determines the loss of kinetic energy associated with each collision.

\section{B. Implementation}

A smoothing function is necessary to translate the mass $m_{i}$ and velocity $\mathbf{v}_{i}$ of the individual grains (indexed by $i$ ) into continuous solid fraction $\rho(x, y)$ and velocity fields $\mathbf{u}(x, y)$ on the grid. A fractional value of $m_{i}$ or $\mathbf{v}_{i}$ is assigned to each of the four neighboring grid nodes and the value is determined by the positional weight of the grain through a linear smoothing function $s\left(\mathbf{r}-\mathbf{r}_{0}\right)$ expressed mathematically as [25]

$$
s\left(\mathbf{r}-\mathbf{r}_{0}\right)= \begin{cases}\left(1-\frac{\Delta x}{l}\right)\left(1-\frac{\Delta y}{l}\right) & \text { if } \Delta x, \Delta y<l \\ 0 & \text { otherwise }\end{cases}
$$

where $\mathbf{r}(x, y)$ is the position of the grain, $\mathbf{r}_{0}\left(x_{0}, y_{0}\right)$ is the position of the grid node, $\Delta x=\left|x-x_{0}\right|$ and $\Delta y=\left|y-y_{0}\right|$ are the relative distances, $l$ is the lattice constant, and $\Sigma_{k} s\left(\mathbf{r}-\mathbf{r}_{k}\right)$ $=1$ with $k$ indexing the four sites. The smoothing of the grains is illustrated in Fig. 3 where nine grid nodes are depicted as red squares on a gradient background. This background is the positional weight map of the central node which is shown as a solid square. From this illustration we observe that all the grains in Fig. 3 make contributions to the field values of the central node: A grain positioned at the central node is assigned a weight of 1 (black background), while a grain positioned along the dotted outline in Fig. 3 is assigned a weight of zero (white background). The weight map, or smoothing function, may also be considered as a collection of equidistant tent functions, distributed over the area in Fig. 3, with maxima rising linearly from zero to 1 and then back to zero. More explicitly, the number density $\rho_{n}$ and average velocity $\mathbf{u}$ associated with a node at position $\mathbf{r}_{0}$ are, respectively, $\rho_{n}\left(\mathbf{r}_{0}\right)=\sum_{i} s\left(\mathbf{r}_{i}-\mathbf{r}_{0}\right)$, and $\mathbf{u}\left(\mathbf{r}_{0}\right)=\sum_{i} \mathbf{v}_{i} s\left(\mathbf{r}_{i}-\mathbf{r}_{0}\right)$, where $i$ runs over the number of particles [25].

The positional weight used to obtain the continuous fields is also used to obtain the pressure forces, $\mathbf{F}_{P}=-\nabla P / \rho_{n}$, 

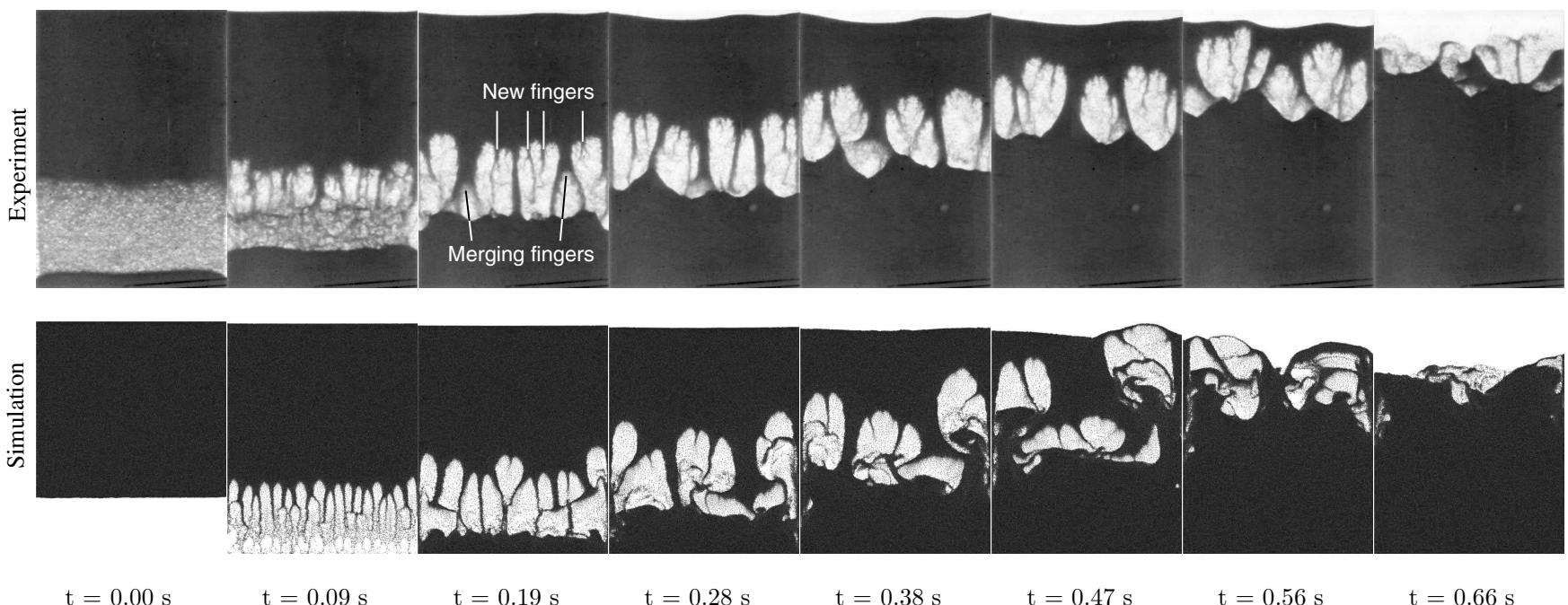

FIG. 4. Images from the experiment (top row) and simulation (bottom row) with polystyrene beads of $140 \mu \mathrm{m}$ in diameter in a closed Hele-Shaw cell that is $56 \mathrm{~mm}$ wide. The experimental cell is $86 \mathrm{~mm}$ high and $1 \mathrm{~mm}$ thick, while the numerical cell is $68 \mathrm{~mm}$ high. The time of the snapshots is the time evolved since the cell reached the upright position. A restitution coefficient of 0.5 is used in the simulations.

acting on the grains. Explicitly for a grain at $\mathbf{r}_{i}, \mathbf{F}_{P}=$ $-\Sigma_{k}\left(\nabla P / \rho_{n}\right)_{k} s\left(\mathbf{r}_{i}-\mathbf{r}_{k}\right)$ with a $k$ index running over the four nodes. In other words, the share of $\mathbf{F}_{P}$ assigned to the grain from the node is equal to the share of $m_{i}$ or $\mathbf{v}_{i}$ assigned to the node from the grain.

A few approximations are made in the implementation of the model. The two-dimensional (2D) solid fraction of disks is translated into a $3 \mathrm{D}$ solid fraction of spheres by multiplying the 2D solid fraction with $2 / 3$, which is the ratio of the $3 \mathrm{D}$ to the $2 \mathrm{D}$ random-close-packed solid fractions. Further, the Carman-Kozeny relation is not valid as the solid fraction approaches zero, and a lower cutoff on the 2D solid fraction, $\rho_{\text {min }}=0.25$, is introduced. These matters are elaborated in Sec. II C of Ref. [25].

The packing of grains used in the simulations is made of 160000 grains with a mean diameter of $140 \mu \mathrm{m}$. This high number of grains is selected to allow a match with the spatial dimensions of the experimental cell. The simulation shown in Fig. 4 took roughly 12 days to complete on a standard PC. Note, however, that the instability emerges as well in simulations with a much more reduced number of grains (e.g., a few thousand). The size distribution adopted for the granular packing is flat and has a $\pm 10 \%$ variation in the diameter. This minor polydispersity is necessary to avoid a hexagonal stacking of the grains.

The granular packing is generated by raining grains from a given height, with random horizontal positions, in a cell without air. After all the grains have settled the packing is allowed to further compactify and relax before the air is introduced. This is to prevent the source term of Eq. (1), $-P \boldsymbol{\nabla} \cdot \mathbf{u}$, to introduce local pockets of overpressure, due to moving grains, that otherwise could perturb the numerical results.

\section{RESULTS}

The rich behavior of the instability provides a number of interesting results which is presented as follows. A qualita- tive discussion and comparison of the experimental and numerical images are given first, followed by solid fraction and pressure profiles from the numerical data in Sec. IV B. A series of investigations, spawned by the Fourier power spectrum of the solid fraction, is presented in Sec. IV C. These results include a quantitative comparison of the experimental and numerical data, in addition to the temporal evolution of the wave numbers obtained from the solid fraction power spectra. Section IV D investigates the response of the instability to changes in the granular dissipation, the initial airgrain interface, and the grain density.

\section{A. Experimental and numerical images}

Figure 4 shows experimental and numerical images of the granular Rayleigh-Taylor instability: A layer of grains displacing a layer of air in a gravitational field. The width of both the experimental and numerical system is $56 \mathrm{~mm}$, while the height of the experimental cell is $86 \mathrm{~mm}$, and the height of the numerical cell is $68 \mathrm{~mm}$. The experimental images are color-inverted and contrast-enhanced versions of the original images; the grains are black and the air is white. The time is set to zero when the cell reaches the vertical position. In the experiment some grains start to fall during the rotation of the cell and a small pile of grains has already formed at the bottom of the first frame.

An air-grain interface, initially flat in the simulation but slightly perturbed in the experiment, is defined by the falling grains. When the cell is flipped a pattern of fine fingers emerges along the interface (see Fig. 5) which subsequently develop into coarser bubble-finger structures that propagate through the packing. A more detailed analysis of the evolution introduces three stages of development: The first stage is the nucleation stage characterized by a transition from a homogeneous decompaction to the appearance of the first finger seeds. This stage is only observed in the numerical snapshots because of the reduced initial resolution of the experimental setup: Transient perturbations disturb the initial 

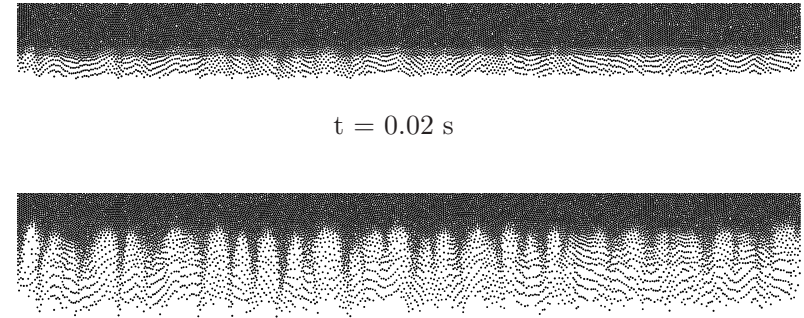

$\mathrm{t}=0.04 \mathrm{~s}$

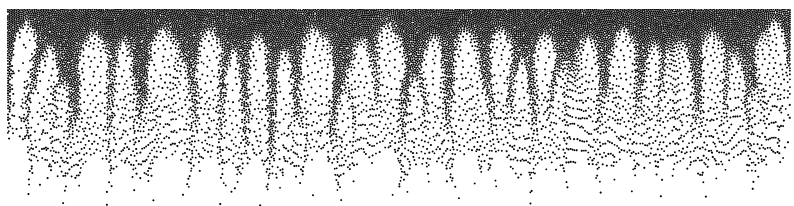

$\mathrm{t}=0.06 \mathrm{~s}$

FIG. 5. A closer look at the initial formation of fingers in the simulation. Before the fingers appear there is a transient phase of homogeneous decompaction. Notice the cusp-shaped geometry of the air-grain interface.

air-grain interface as the rotating cell suddenly stops. The second stage is the growing stage where the nucleated fingers grow in size and start to coalesce with their neighboring fingers. The third and final stage is the propagation stage, which is recognized by the aggregation of grains at the bottom of the cell and the formation of bubbles that propagate through the packing. Two competing mechanisms are at play here, one producing finer structures, the other producing coarser structures. The coarsening mechanism causes fingers to merge as two fingers form an inverted Y (see the experimental image at $t=0.19$ in Fig. 4). The smaller bubble trapped beneath the wedge of the inverted Y continuously lags behind the bigger bubbles until the small bubble disappears. Thus, two fingers have been reduced to one. The other mechanism, giving rise to finer structures, is manifested as thin filaments forming inside the rising bubbles. These new fingers split the bubbles and prevent them from growing indefinitely.

Figure 5 shows a more detailed series of numerical snapshots of the early evolution of the instability: In the top frame the initial homogeneous decompaction of the air-grain interface is visible. By close comparison of the frames it is possible to discern tiny finger seeds in the top frame that has developed into well-defined fingers in the second and third frames. From the second to the third frame the fingers mainly grow in size and only a few new fingers are nucleated. The first and second frame belongs to the nucleation stage, while the third frame is taken from the growing stage.

Figure 6 shows a snapshot from a simulation where glass beads of $210 \mu \mathrm{m}$ are used. The beads are colored in bands in their initial configuration to illustrate the dynamics of the mixing and the patterns created by the propagating bubbles. The pattern that emerges is reminiscent of geological patterns observed when, e.g., water is forced through sediments of sand [35].

A qualitative comparison of the image series in Fig. 4 renders the simulation and experiment consistent in many

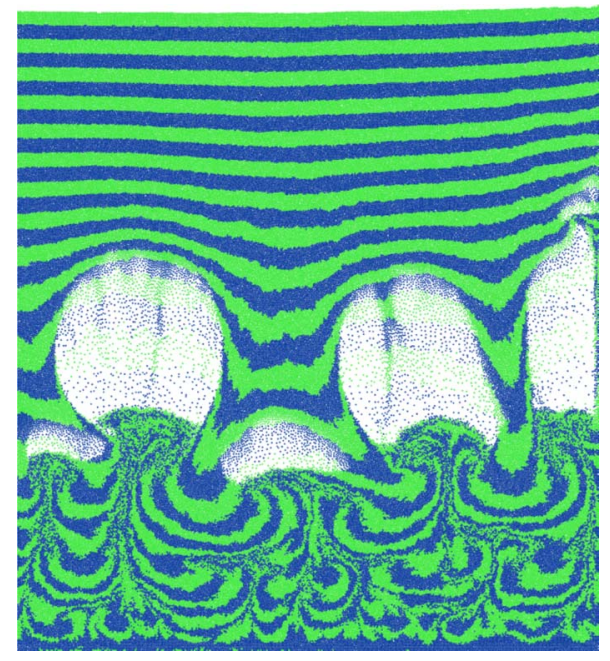

FIG. 6. (Color online) A snapshot from a simulation using glass beads of $210 \mu \mathrm{m}$ in diameter where the initial configuration is colored in horizontal bands to bring out the dynamics of the structures as the instability evolves.

respects. The sizes of the bubbles and the fingers are comparable, and the dynamical processes of finger merging and finger nucleation are observed in both cases. Nevertheless, some discrepancies are observed, particularly at the start and towards the end of the instability: The initial decompaction, followed by the emergence of the first fine fingers, is only observed numerically. Towards the end of the process a horizontal destabilization of the air-grain interface is visible, i.e., some bubbles reach the surface before others.

The initial discrepancies can to a large extent be explained by three experimental features: The increased thickness of the cell allowing up to seven layers of grains between the plates, the rotation of the cell, and the abrupt stop of the cell as it reaches the vertical. Ideally the rotation would be so fast that the grains did not move at all during the rotation. In the experiment this is, however, not the case and the grains will slide and roll down the inclined plate during the rotation while the air passes in a channel above the grains (known as the granular Boycott effect [19]). Only when the cell is almost vertical is the sliding layer of grains able to form an interface that fills the whole cross section of the cell so that fingers may start to form. However, a mechanical shock is created in the cell as it hits the bar. The effect is a transverse oscillation that causes the grains to be tossed back and forth between the plates. Any fingers formed prior to the shock will be strongly perturbed and even erased. The result is visible in the first experimental image of Fig. 4 where the falling grains form an almost homogeneous sheet of grains rather than well-defined fingers.

Some differences are also found in the shape of the fingers: In the numerical images the fingers appear somewhat buckled and bended compared to the experimental fingers. We believe this is an artifact caused by the cutoff imposed on the solid fraction. Since the solid fraction is not allowed to be less than 0.25 , the empty space porosity, i.e., the porosity in a region with no or very few grains, will be different from 1. This again leads to overestimated pressure gradients in the 
most dilute regions of the system, and forces are exerted on the fingers traversing the gap of air. Nevertheless, the shape of the interface is very well reproduced by the simulations despite the buckling of the fingers.

The experimental instability seems to be more stabilized horizontally than the numerical instability. By comparing images in Fig. 4 for $t>0.4 \mathrm{~s}$ it is evident that on a detailed level the numerical images are quite different from the corresponding experimental images. It seems that the most advanced bubble in the numerical images departs from the other bubbles at an increasing rate, causing a horizontal destabilization of the interface. We believe that the reason for this is found in the zero particle-particle friction and, more importantly, the zero particle-wall friction of the numerical model. The friction between the glass plates and the grains in the experiment is probably important in the dense part of the packing, due to the Janssen effect [36]. This has most likely a stabilizing effect on the propagation of the interface. However, apart from this small difference in the propagation of the bubbles, the other quantitative features of the instability are well reproduced by our friction-free simulations. Thus, for the sake of simplicity, we have chosen to discard friction in the model. Indeed, taking friction into account would imply important numerical costs: The addition of two degrees of freedom to account for the rotation of the grains, and a new fitting parameter for the friction coefficient.

Another effect of zero friction in the simulations is observed as the rightmost bubble in the latter numerical images of Fig. 4 reaches the upper surface at about $t=0.45 \mathrm{~s}$ and leaves behind an open air-filled void. As grains rush in to fill this void convection rolls are set up that will distort and perturb the present fingers. Such convection rolls would to a large extent be dissipated away if interparticle friction were present in the simulations.

\section{B. Pressure and solid fraction in the simulation}

The temporal evolution of the pressure $P$ and the solid fraction $\rho$ in the simulation are respectively shown in Figs. 7 and 8 . The vertical profiles $P(y)$ and $\rho(y)$ shown for different times are calculated by averaging $P(x, y)$ and $\rho(x, y)$ over the horizontal component $x$. Figure 7(a) provides a detailed temporal description of $P(y)$ immediately after the rotation of the cell: The initially flat profile is, within a few milliseconds, transformed into a linearly decreasing function while an overpressure builds up at the bottom of the cell. This corresponds to a fast diffusion of the pressure field [cf. Eq. (1)], and shows the transient curvature of the pressure profile relaxing towards a linear function. This happens at a time scale $\ell^{2} /(P \ell \kappa / \mu) \simeq 0.1 \mathrm{~ms}$, smaller than the time scale associated with grain motion due to gravity, $\sqrt{d / g} \simeq 3 \mathrm{~ms}$, where $\ell$ is the system size, and $d$ the grain diameter. Figure 7(b) shows $P(y)$ for later times, and the linearity of the pressure in the upper part of the packing, above the air-grain interface, is almost unchanged. Figure 8 shows $\rho(y)$ for the same times as in Fig. 7(b). The initially sharp air-grain interface is smeared out as the grains fall and subsequently fill up the bottom of the cell.

Figure 9(a) shows the temporal evolution of the pressure at the bottom of the cell, i.e., $P(y=0, t)$. Figure $9(\mathrm{~b})$ shows
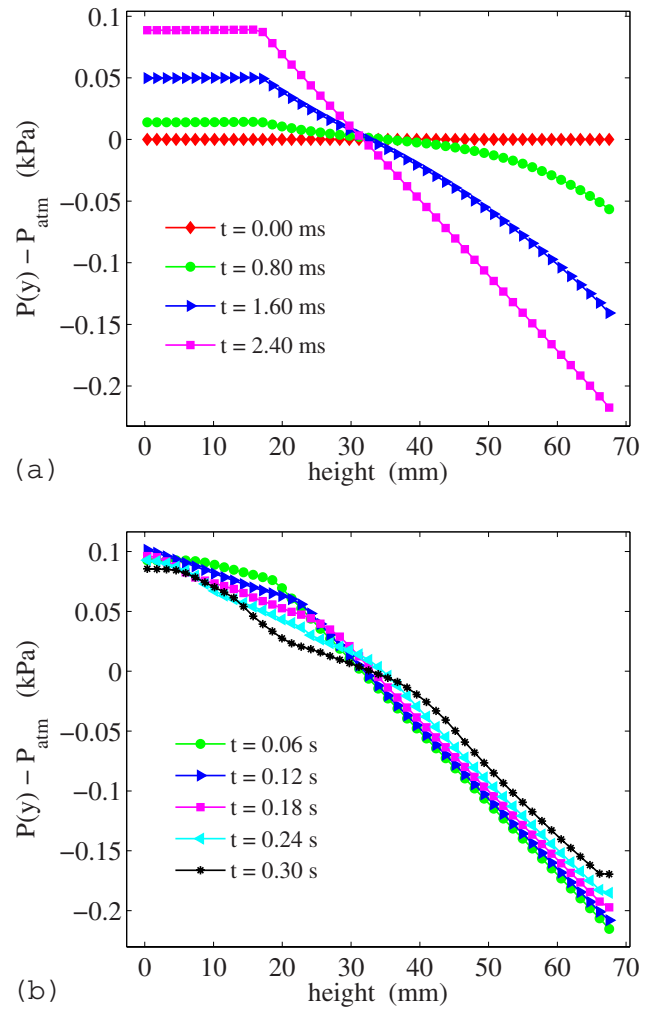

FIG. 7. (Color online) Plots of vertical pressure profiles averaged over the system width. (a) Evolution of the pressure immediately after the cell is rotated. (b) The pressure profiles at later times. The pressure is given as the deviation from $1 \mathrm{~atm}$.

the averaged vertical velocity of the whole packing, $\left\langle v_{y}(t)\right\rangle$ (average of $v_{y}$ for all the grains). Due to the compressibility of the air the pressure is observed to undergo a transient damped oscillation immediately after the cell is rotated. The rotation of the numerical cell is instantaneous, in contrast to the rotation of the experimental cell, and the sudden acceleration of the grains generates a pressure shock front that propagates into the gap of air. The oscillating pressure causes the averaged vertical velocity of the grains to display a similar oscillation as shown in Fig. 9(b).

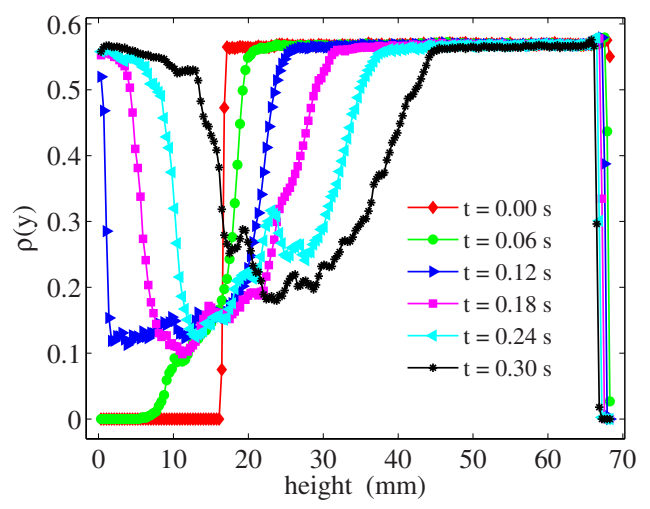

FIG. 8. (Color online) Plot of vertical solid fraction profiles averaged over the system width. The times of the profiles, except for the first profile, coincide with the times in Fig. 7(b). 

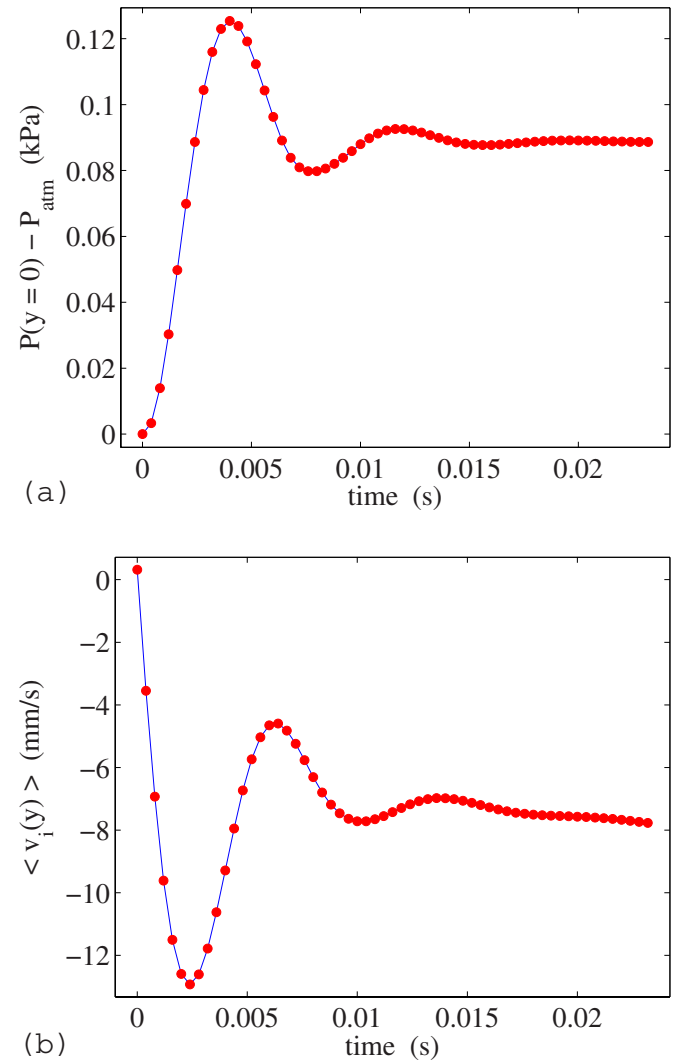

FIG. 9. (Color online) (a) Temporal evolution of the pressure at the bottom of the cell. (b) Vertical velocity of the packing calculated by averaging over all the grains. Immediately after the cell is flipped a damped oscillation is observed in both pressure and velocity, which is a signature of the compressibility of the air.

\section{Fourier analysis}

\section{Solid fraction}

In addition to the qualitative comparison of the numerical and experimental data, a quantitative validification is performed by means of spatial Fourier power spectra of the solid fraction. By this analysis an average wave number $\langle k\rangle$ is obtained which measures the characteristic inverse size of the observed structures.

The average wave number $\langle k\rangle$ is calculated as follows. The power spectrum $S(k ; j)$ of the solid fraction is calculated by applying the discrete Fourier transform [37] on the horizontal lines $j$ of the solid fraction field $\rho(x, y)$. Here $k$ denotes the wave numbers of $\rho(x ; j)$, and $j$ is an index running over the horizontal lines of the solid fraction grid. The power spectra $S(k ; j)$ are further averaged over $j$ to produce a single power spectrum $\bar{S}(k)$, representing the state of the system at a given time. The mean $\langle k\rangle$ of the averaged power spectrum $\bar{S}(k)$ is then finally obtained by the common definition of the $n$th moment

$$
\left\langle k^{n}\right\rangle=\frac{\sum k^{n} \bar{S}(k)}{\sum \bar{S}(k)}
$$

with $n=1$. The standard deviation
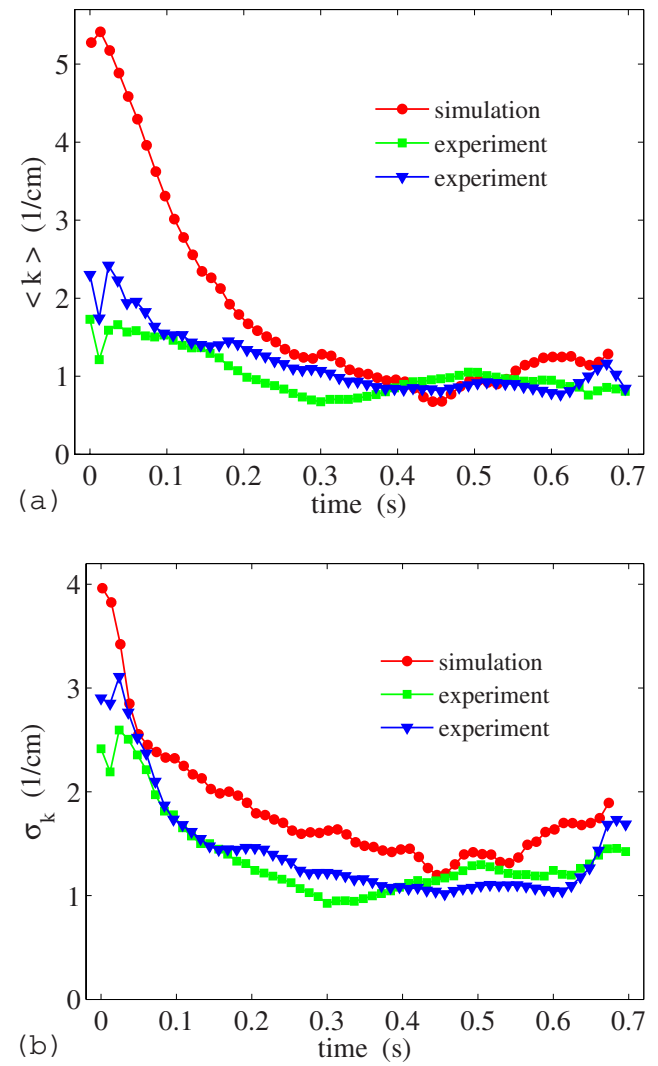

FIG. 10. (Color online) (a) Mean wave number $\langle k\rangle$ of $\bar{S}(k)$ as function of time. (b) Standard deviation $\sigma_{k}$ of $\bar{S}(k)$ as function of time. The results are obtained for two experiments and one simulation, all using polystyrene beads of $140 \mu \mathrm{m}$ in diameter.

$$
\sigma_{k}=\sqrt{\left\langle k^{2}\right\rangle-\langle k\rangle^{2}}
$$

is also calculated. The unit of $\langle k\rangle$ is $\mathrm{cm}^{-1}$, and it is the characteristic inverse length scale of the observed structures.

The same analysis is used to extract information about the characteristic size of the experimental structures. The solid fraction is however not directly accessible in the experimental data, and the values of the image pixels, spanning from 0 (black) to 255 (white), are instead used as an estimate of the real solid fraction. The solid fraction and the pixel value are inverse quantities, i.e., dilute regions have low solid fractions but high pixel values since they appear as white in the images.

The experimental images are 322 pixels in width, whereas the numerical solid fraction grid is only 160 nodes in width. If the mean values of the two power spectra are to be comparable, the width of the two distributions must be equal. Hence, the experimental power spectrum has an upper cutoff given by the largest wave number in the numerical power spectrum.

The mean, $\langle k\rangle$, and standard deviation, $\sigma_{k}$, of the power spectrum are plotted as functions of time in Figs. 10(a) and $10(\mathrm{~b})$, respectively. The data used here are the same as in Fig. 4, with an additional data-set from a similar experiment. As the instability evolves the initial discrepancy is reduced, and the consistency of the data for later times is quite good. 

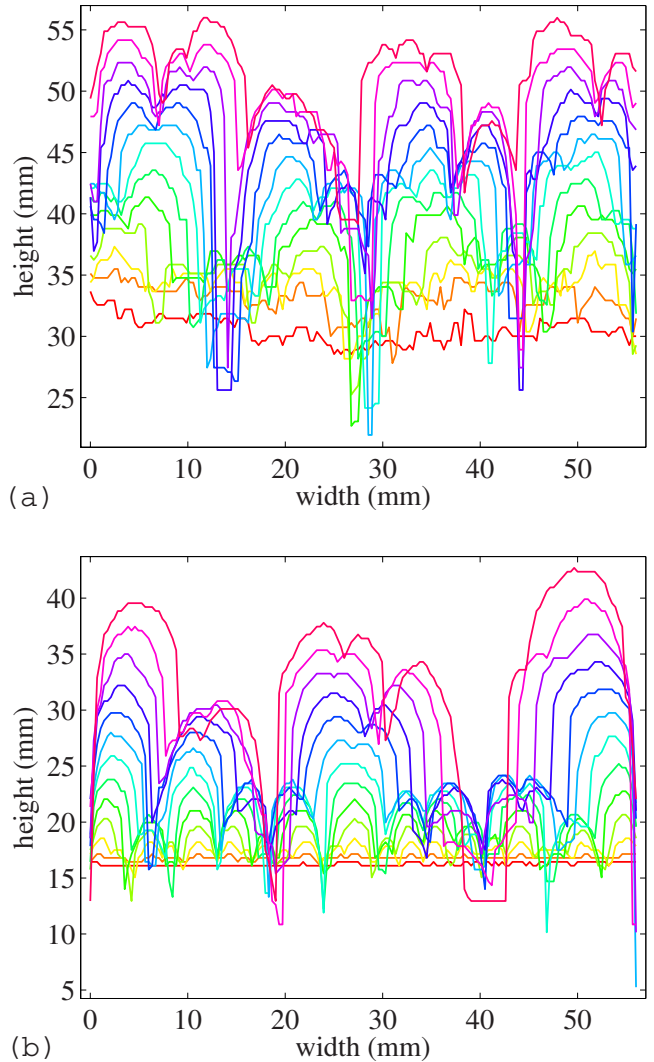

FIG. 11. (Color online) Temporal evolution of (a) the experimental air-grain interface and (b) the numerical air-grain interface. The interfaces move upward with a temporal separation of $0.024 \mathrm{~s}$. The times of the first and last interface are, respectively, 0.002 and $0.29 \mathrm{~s}$.

The decrease of $\sigma_{k}$ in Fig. 10(b) is caused by the increasing length of the fingers: As the fingers grow the amplitude of the wave number associated with the finger separation increases, thus reducing the width of the power spectrum distribution.

These results show that our gas-grain system is clearly different from the liquid-grain system discussed by Völtz et al. [21]. Their liquid-grain system did not display a wave number shift with time, nor the cusp-shaped geometry of the fingers.

\section{The interface}

Instead of analyzing the full solid fraction field $\rho(x, y)$ for the whole system as in the previous section, the focus is now on the dynamics of the air-grain interface itself.

The function describing the interface is determined by the following procedure. Each vertical line of the solid fraction grid is scanned from top to bottom, and the first node on the line with a solid fraction less than a given threshold is defined to be a point on the interface. Together these points define the interface. The value of the threshold is determined by plotting the calculated interfaces on top of the numerical snapshots and visually confirm that they correspond well (see Fig. 2 of Ref. [20]). The overall shape of the interface is not very sensitive to a fine-tuning of the threshold. The ex-
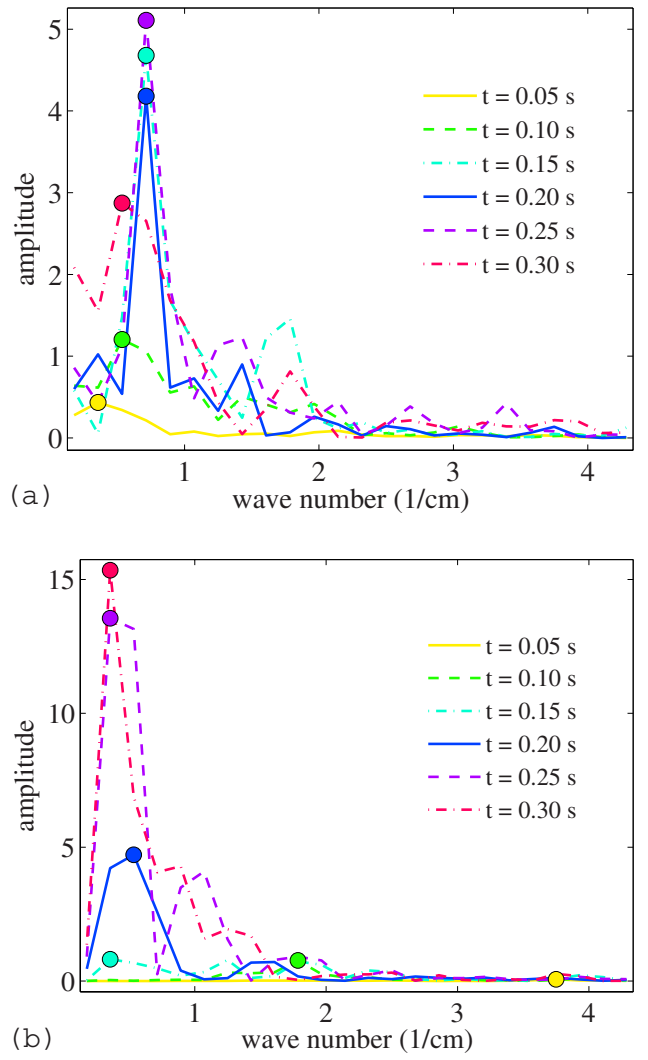

FIG. 12. (Color online) Temporal evolution of (a) the experimental power spectrum and (b) the numerical power spectrum. The power spectrum of every second interface in Fig. 11 is plotted here. The times of the power spectra are given in the legend box, and the colored circles indicate the location of the maximum wave number for every time.

perimental interface is obtained by the same procedure, only with the solid fraction replaced by the image pixel values. The experimental images are rescaled to the size of the numerical solid fraction grid before the experimental interface is obtained. The resulting single-valued functions describing the interface at different times are shown in Figs. 11(a) and 11(b) for the experiment and simulation, respectively. The two series of interfaces have the same temporal separation of $24 \mathrm{~ms}$, and they both start (bottom) at $t=0.002 \mathrm{~s}$ and stop (top) at $t=0.29 \mathrm{~s}$. Salient features of these front evolution plots are the expansion of the bubbles and the appearance of new fingers (shaped like wedges in these plots) at the top of the latter interfaces. Comparing Figs. 11(a) and 11(b), the final shape of the interfaces is in good agreement. The initial shape of the interfaces do not coincide equally well due to the external disturbances introduced in the experimental cell.

Figures 12(a) and 12(b) show (in the same colors as in Fig. 11) the power spectrum of every second interface in Figs. 11(a) and 11(b), respectively. The location of the maximum wave number for each individual power spectrum is indicated by circles. The numerical power spectra in Fig. 12(b) starts out with a maximum wave number to the far right, whereas the experimental power spectra in Fig. 12(a) starts out with a maximum wave number to the far left. The reason for this discrepant behavior is the very different shape of the initial experimental and numerical interfaces: The nu- 

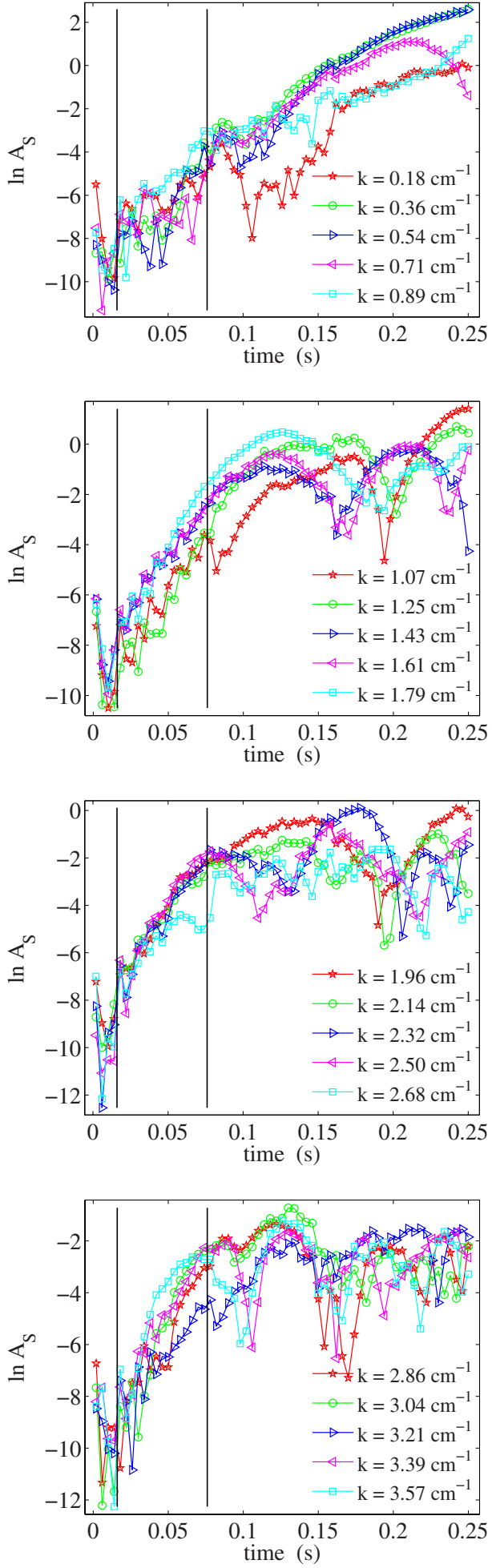

FIG. 13. (Color online) Growth rates of the air-grain interface. The wave numbers are given in the legend boxes, and the vertical lines indicate the time window where the dispersion relation in Fig. 15(a) is calculated.

merical interface is virtually flat, whereas the experimental interface has noise on all wavelengths. The dominating wave numbers of the latter power spectra in Figs. 12(a) and 12(b) are only slightly shifted with respect to each other, and converge to approximately the same form.
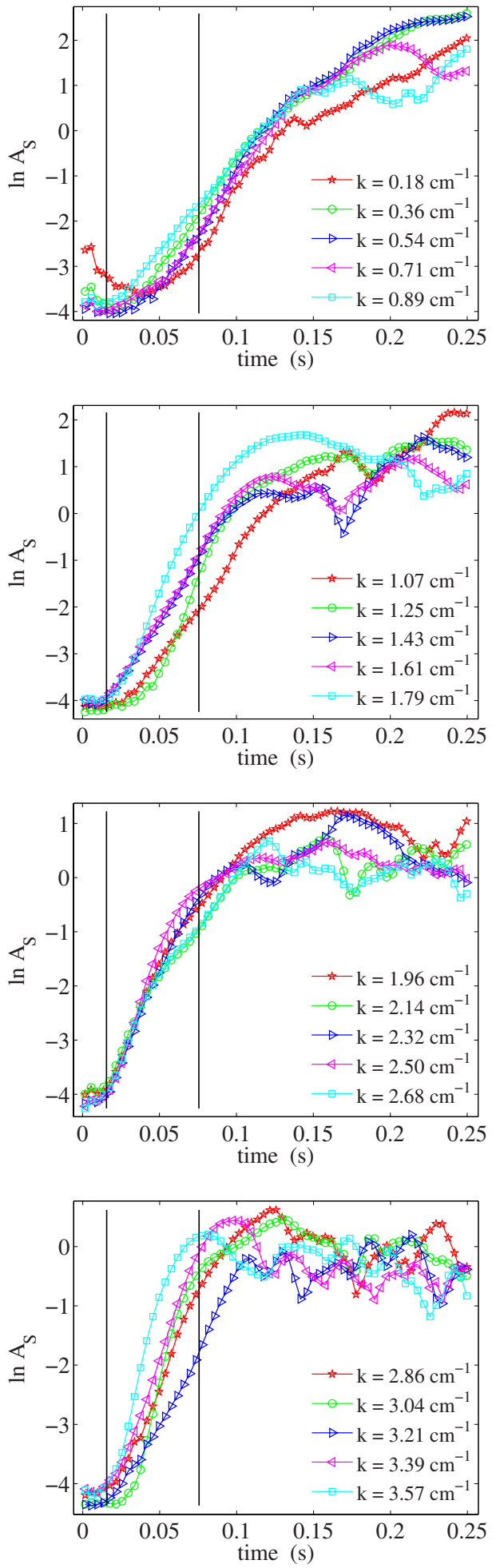

FIG. 14. (Color online) Growth rates of the bulk solid fraction. The wave numbers are given in the legend boxes, and the vertical lines indicate the time window where the dispersion relation in Fig. 15 (b) is calculated.

\section{Growth rates}

We now move on to analyze the growth rates of the wave numbers as they appear in the power spectra of the numerical data. Following the lines of a linear stability analysis we look 

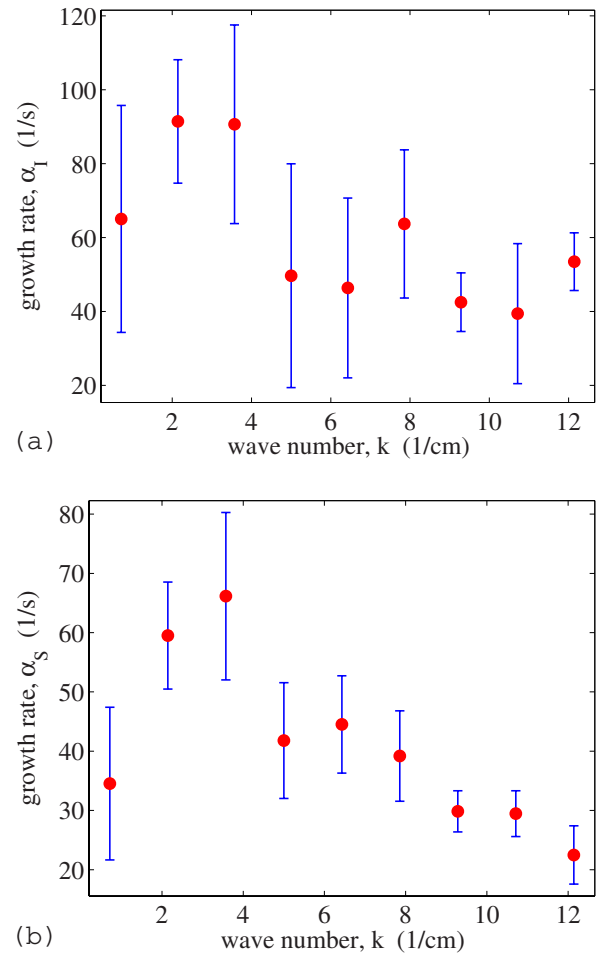

FIG. 15. (Color online) Dispersion relation (a) of the air-grain interface and (b) for the bulk solid fraction. The relations are obtained by linear fits over the approximate linear parts of the growth rate plots in Figs. 13 (interface) and 15 (bulk solid fraction). The error bars are the standard deviations within the boxes.

for possible early exponential growth of the wave numbers in the power spectrum of both the air-grain interface profile and the full solid fraction field.

The temporal evolution of the 20 smallest wave numbers in the power spectrum (or equivalently the 20 longest wavelengths) of the interface profile are plotted semilogarithmically in Fig. 13. The corresponding plots for the average power spectrum of the solid fraction field, $\bar{S}(k)$, are shown in Fig. 14. Exponentially growing wave numbers will appear as linear in these plots. The growth of the solid fraction wave numbers in Fig. 14 is less noisy compared to the growth of the interface wave numbers in Fig. 13 due to the averaging of the solid fraction power spectrum $\bar{S}(k)$ (see IV C 1 ).

Judging by the growth rates shown in Figs. 13 and 14 it seems fair to conclude that none of the wave numbers follow an exponential growth (at least not of any significant duration). Hence, the granular Rayleigh-Taylor instability is inherently nonlinear, in contrast to the classical RayleighTaylor instability. Nevertheless, a linear least squares fit is obtained for all the wave numbers of the power spectrum, including those not displayed in Figs. 13 and 14. The fit is performed over a time window of $0.6 \mathrm{~s}$ as indicated in the plots. The resulting growth rates $\alpha_{I}$ (interface) and $\alpha_{S}$ (solid fraction) as functions of wave number $k$ are shown in Figs. 15(a) and 15(b), respectively. In these figures each data point is a box average over eight wave numbers. The standard deviation is indicated by the error bars. Given the approximate linearity of the wave number growth, vigorous conclu- sions cannot be drawn from these plots. However, while our measurements do not serve to identify a well defined window of linear growth, the growth rates nevertheless exhibit the dominating, time dependent wave number: The maxima at $\sim 4 \mathrm{~cm}^{-1}$ in Figs. 15(a) and 15(b) coincide with the $t$ $=0.05 \mathrm{~s}$ maximum of the power spectrum in Fig. 12(b). However, it is clear that nonlinear mechanisms govern the selection of the final dominating wavelength.

In a well-controlled experiment of the classical RayleighTaylor instability it is possible to observe exponential growth over a time sufficiently long to erase the influence of the initial noise. This is not the case, however, for the granular Rayleigh-Taylor instability since the exponential domain of the wave number growth is too short to cancel the initial noise. Hence, the structures that develop early, i.e., on a time scale of about $0.1 \mathrm{~s}$, are sensitive to the initial structure of the system. Note that this initial structure is not an experimental imperfection but an intrinsic, unavoidable feature of granular packings.

\section{Changing simulation parameters}

A number of simulations are performed to probe the response of the instability as the input parameters are changed, i.e., the density of the grains, the roughness of the initial interface, and the dissipation of the granular phase.

\section{Grain density}

A comparison of simulations performed using grains of different densities, but with identical start configurations, is presented. The density of the grains is $1.05 \mathrm{~g} / \mathrm{cm}^{3}$ (polystyrene) or $2.5 \mathrm{~g} / \mathrm{cm}^{3}$ (glass), and the diameter is $140 \mu \mathrm{m}$. Figures 16(a) and 16(b) show plots of the mean wave number $\langle k\rangle$ and the standard deviation $\sigma_{k}$ of the power spectrum $\bar{S}(k)$ as functions of time. From these plots it is observed that the time of breakthrough, i.e., when the bubbles reach the upper surface, is dependent on the density of the grains. The separation in time between the last data points of each curve in Fig. 16(a) shows that the instability develops faster if heavier beads are used. However, the initial decrease (i.e., from $t$ $=0$ to $0.1 \mathrm{~s})$ of $\langle k\rangle$ and $\sigma_{k}$ are roughly the same for the two densities.

\section{Initial interface}

The response of the instability to different initial air-grain interfaces is investigated by three simulations using interfaces with a variation in the roughness.

The three interfaces, shown in Fig. 18 where they are denoted $A, B$, and $C$ in increasing order of roughness, are prepared as follows. Starting from a configuration where the packing rests on the bottom plate, interface $A$ is created by displacing the packing vertically upward to fill the top half of the cell. In this case the cell is not rotated. A perfectly flat air-grain interface is now defined by the grains originally positioned on the bottom plate, prior to the vertical displacement. Interface $B$ is created by first removing all grains with their centers above a given height just below the upper surface of the packing (again positioned on the bottom plate). 

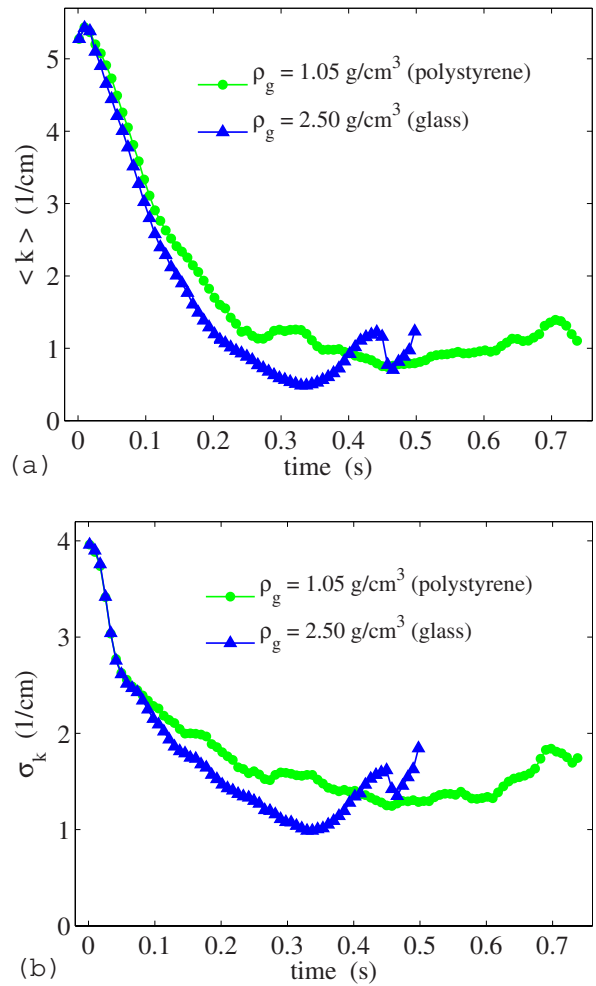

FIG. 16. (Color online) (a) Mean wave number $\langle k\rangle$ as a function of time for different grain densities. (b) Standard deviation $\sigma_{k}$ as a function of time for different grain densities. The grain densities $\rho_{g}$ used in the simulations are given in the legend box.

The cell is then rotated to bring the slightly rough surface above the layer of air. Interface $C$ is the rotated original packing with a surface determined by the local equilibrium of the grains.

The temporal evolution of $\langle k\rangle$ and $\sigma_{k}$ for packings with their initial interfaces as shown in Fig. 18 are plotted in Figs. 17 (a) and 17(b), respectively. These plots lead to the conclusion that the shape of the initial interface has an effect only during the earliest stages of the instability. It appears that the slowest evolving interface is $B$, and that $B$, not $A$, is the interface with the smallest initial noise. This may correspond to the fact that the porosity above interface $A$ has larger fluctuations than in $B$ because of the constraint imposed by the flat wall. After about $0.2 \mathrm{~s}$ the difference in $\langle k\rangle$ is negligible. The increased standard deviation of interface $A$ indicates a slightly slower and delayed generation of fingers in this case. However, the effect is only temporary and the initial difference is canceled out. Note that this observation is consistent with the conclusion at the end of Sec. IV C 3: The exponential domain is too short to cancel the initial noise during the incipient evolution of the instability. However, as nonlinear effects take over, the system nevertheless evolves toward structures that are insensitive to the initial noise.

\section{Dissipation}

A series of simulations are performed where the coefficient of normal restitution, $\varepsilon$, is changed. The motivation is to investigate the effect on the fingering process and its dy-
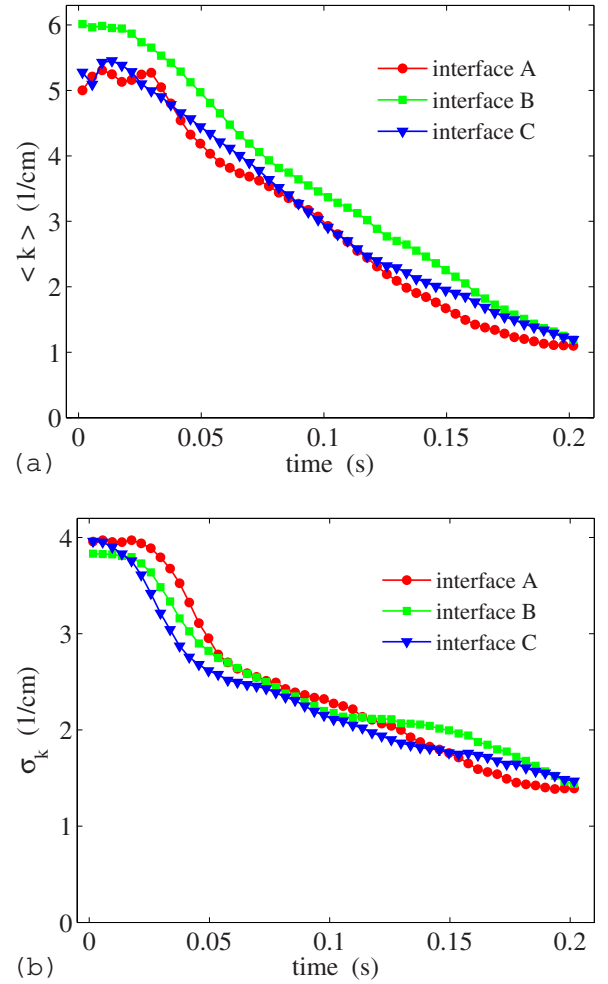

FIG. 17. (Color online) (a) Mean wave number $\langle k\rangle$ as function of time for different initial interfaces. (b) Standard deviation $\sigma_{k}$ as function of time for different initial interfaces. The interfaces used in the simulations are labeled $A, B$, and $C$ and are shown in Fig. 18.

namics. Figure 19 shows two snapshots for each of the six different restitution coefficient used in these simulations. The two leftmost snapshots in Fig. 19, with $\varepsilon=1.0$ and 0.95, distinguish themselves by a diffuse outline of the interface, fewer fingers, and a general lack of detail compared to the other snapshots. To differentiate between the remaining pairs of snapshots, obtained with $\varepsilon=0.8,0.5,0.2$, and 0.05 , is not as evident because it is difficult to identify significant differences in these images: The number of fingers is about the same, as well as the size and shape of the bubbles. It is possible to identify minor deviations in, e.g., the number and shape of the renucleated fingers, but no significant influence of dissipation is observed on the formation of new fingers. The conclusion must be that the system is invariant under changes in the dissipation as long as the restitution coefficient is below a certain threshold. However, above this threshold the instability is sensitive to variations in the restitution, in particular the size and shape of the fingers are affected. The curvature of the bubbles is only moderately affected by the variation in $\varepsilon$.

The value of the threshold, above which the instability is virtually insensitive to variations in $\varepsilon$, is likely to depend on

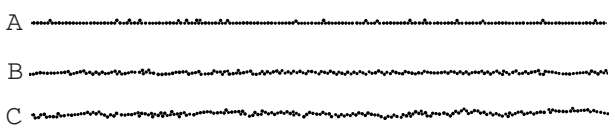

FIG. 18. Different initial air-grain interfaces used in the simulations. The interfaces are labeled $A, B$, and $C$ in increasing order of roughness. 

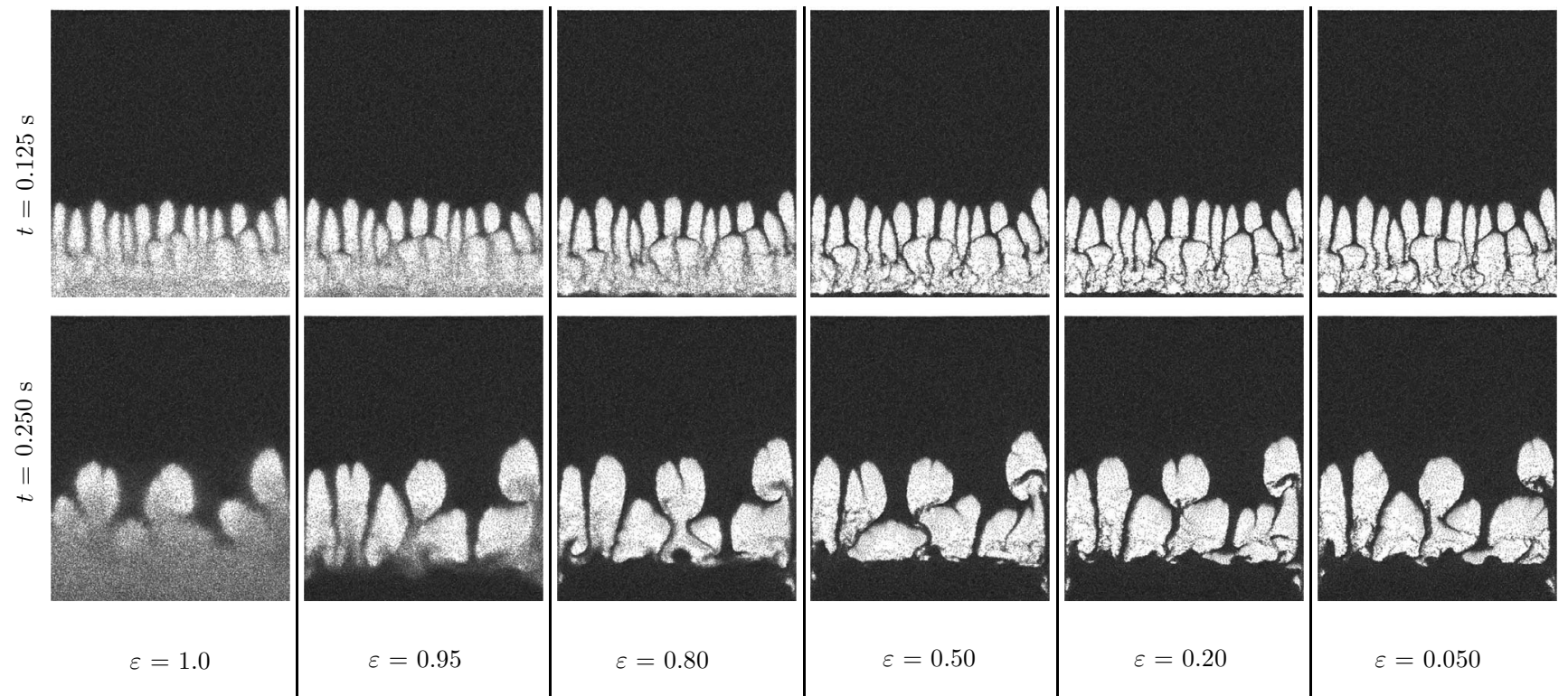

FIG. 19. Snapshots of simulations with different coefficients of restitution $\varepsilon$, given below the snapshots. The times of the simultaneous snapshots are given to the left of each row.

the average number of collisions the grains participate in. The number of collisions $n$ required to reduce the kinetic energy by a given fraction $F$ is inversely proportional to the logarithm of the restitution coefficient $\varepsilon$. In mathematical terms $n(\varepsilon)=\ln (1-F) / \ln (\varepsilon)$ which follows from the relation $\varepsilon^{n}=1-F$. A semilogarithmic plot of $n(\varepsilon)$ for three different values of $F$ is shown in Fig. 20. Lets assume a grain has lost $90 \%$ of its kinetic energy by interacting in $n^{\prime}$ collisions with restitution coefficient $\varepsilon^{\prime}$. If the restitution coefficient is reduced beyond $\varepsilon^{\prime}$ it will make only a negligible difference because the energy was already very small at $\varepsilon^{\prime}$. Figure 20 demonstrates that the number of collisions needed to reduce the energy by, e.g., $90 \%$ changes significantly as the restitution coefficient changes from 0.99 ( 229 collisions) to 0.8 (ten collisions). Below this interval the change in the number of collisions is marginal: At $\varepsilon=0.6$ it takes five collisions to

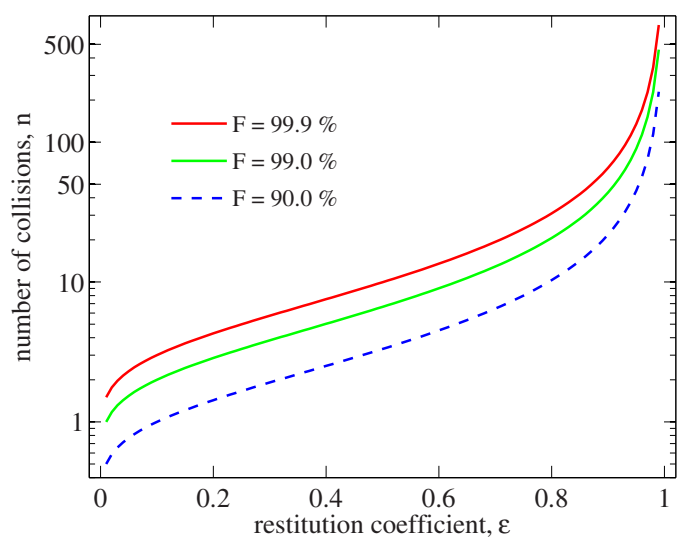

FIG. 20. (Color online) Number of collisions $n$ as a function of restitution coefficient $\varepsilon$ (normal). The three different curves show how many collisions it takes to reduce the kinetic energy of a grain by a fraction $F$ (given in the legend box) for different $\varepsilon$. reduce the energy by $90 \%$, compared to ten collisions at $\varepsilon$ $=0.8$. If we assume that a grain on average is involved in ten collisions, over a time scale that is small compared to the dynamical time scale of the structures, $90 \%$ of its energy is lost if $\varepsilon=0.8$, and $99 \%$ is lost if $\varepsilon=0.6$. Since most of the energy is already dissipated at $\varepsilon=0.8$, this might explain why the simulations are virtually invariant if the restitution coefficient is reduced beyond $\varepsilon=0.8$.

\section{CONCLUSION}

A gravitational instability, observed as a layer of dense granular material is positioned above a layer of air in a closed Hele-Shaw cell, is reported both experimentally and numerically. Qualitative and quantitative comparisons of numerical and experimental data are presented with the conclusion that the simulations reproduce the essential experimental features. The growth rates of the numerical solid fraction field are obtained by Fourier analysis to look for exponential time dependence. Both the bulk solid fraction and the airgrain interface have been investigated by this analysis. Our conclusion is that granular Rayleigh-Taylor instability is inherently nonlinear since exponential dependence of significant duration is not observed in the data. Further, the response to changes in different simulation parameters was probed: The density of the grains, the initial air-grain interface, and the granular dissipation. Increasing the density leads to an earlier breakthrough of the air bubbles, without any significant change in the coarsening time of the structures. Changing the roughness of the initial interface has an effect only early in the instability; the difference is canceled after about $0.2 \mathrm{~s}$. Dissipation affects only the formation of fingers above a certain threshold of the restitution coefficient. 
This behavior is supported by a simple model for the loss of granular kinetic energy.

The unique behavior of the gas-grain system as compared to the liquid-grain system investigated by Völtz et al. might be caused by the compressibility and low inertia of the air.

As for the experimental work, some refinements of the experiment is necessary if the complete evolution of the instability, including the initial fingers, is to be probed.

\section{ACKNOWLEDGMENTS}

The work was supported by NFR, the Norwegian Research Council. A special thanks to Sean McNamara for useful comments, and for making the original F90 implementation of the gas-grain model available during the $\mathrm{C}++$ reimplementation.
[1] Physics of Dry Granular Media, edited by H. J. Herrmann J.-P. Hovi, and S. Luding, NATO Advanced Study Institute, Series E: Applied Sciences (Kluwer Academic Publishers, Dordrecht, 1998), Vol. 350.

[2] J. F. Davidson and D. Harrison, Fluidization (Academic Press, New York, 1971).

[3] D. Gidaspow, Multiphase Flow and Fluidization (Academic Press, San Diego, 1994).

[4] G. K. Batchelor, J. Fluid Mech. 52, 245 (1972).

[5] J. F. Richardson and W. N. Zaki, Trans. Inst. Chem. Eng. 3, 65 (1954).

[6] P. Y. Julien, River Mechanics (Cambridge University Press, Cambridge, U.K., 2002).

[7] D. H. Rothman, J. P. Grotzinger, and P. B. Flemings, J. Sediment Res. 64, 59 (1994).

[8] R. Bachrach, A. Nur, and A. Agnon, J. Geophys. Res. 106, 13515 (2001).

[9] P. G. Drazin and W. H. Reid, Hydrodynamic Stability (Cambridge University Press, Cambridge, U.K., 1981).

[10] S. Chandrasekhar, Hydrodynamic and Hydromagnetic Stability (Dover Publications, New York, 1981).

[11] P. G. Saffman and G. Taylor, Proc. R. Soc. London, Ser. A 245, 312 (1958).

[12] C. Chevalier, M. B. Amar, D. Bonn, and A. Lindner, J. Fluid Mech. 552, 83 (2006).

[13] Ø. Johnsen, R. Toussaint, K. J. Måløy, and E. G. Flekkøy, Phys. Rev. E 74, 011301 (2006).

[14] G. I. Taylor, Proc. R. Soc. London, Ser. A 132, 499 (1931).

[15] M. P. Ciamarra, A. Coniglio, and M. Nicodemi, J. Phys.: Condens. Matter 17, 2549 (2005).

[16] J. J. Wylie, Q. Zhang, and X. Sun, Phys. Rev. Lett. 97, 104501 (2006).

[17] F. Malloggi, J. Lanuza, B. Andreotti, and E. Clement, Europhys. Lett. 75, 825 (2006).

[18] D. Gendron, H. Troadec, K. J. Måløy, and E. G. Flekkøy, Phys. Rev. E 64, 021509 (2001).
[19] E. G. Flekkøy, S. McNamara, K. J. Måløy, and D. Gendron, Phys. Rev. Lett. 87, 134302 (2001).

[20] J. L. Vinningland, Ø. Johnsen, E. G. Flekkøy, R. Toussaint, and K. J. Måløy, Phys. Rev. Lett. 99, 048001 (2007).

[21] C. Völtz, W. Pesch, and I. Rehberg, Phys. Rev. E 65, 011404 (2001).

[22] G. Taylor, Proc. R. Soc. London, Ser. A 201, 192 (1950).

[23] R. Jackson, The Dynamics of Fluidized Particles (Cambridge University Press, Cambridge, U.K., 2000).

[24] S. Schwarzer, Phys. Rev. E 52, 6461 (1995).

[25] S. McNamara, E. G. Flekkøy, and K. J. Måløy, Phys. Rev. E 61, 4054 (2000).

[26] D.-V. Anghel, M. Strauss, S. McNamara, E. G. Flekkøy, and K. J. Måløy, Phys. Rev. E 74, 029906(E) (2006).

[27] H. Darcy, Les Fontaines Publiques de la Ville de Dijon (Dalmont, Paris, 1856).

[28] P. C. Carman, Trans. Inst. Chem. Eng. 15, 150 (1937).

[29] F. Radjai, M. Jean, J.-J. Moreau, and S. Roux, Phys. Rev. Lett. 77, 274 (1996).

[30] L. Brendel and S. Dippel, Physics of Dry Granular Media (Kluwer Academic, Dordrecht, 1998).

[31] M. P. Allen and D. J. Tildesley, Computer Simulation of Liquids (Clarendon Press, Oxford, 1989).

[32] J. J. Moreau, Eur. J. Mech. A/Solids 13, 93 (1994).

[33] M. Jean, in Mechanics of Geometrical Interfaces, edited by A. P. S. Selvadurai and M. J. Boulon (Elsevier Sciencs, Amsterdam, 1995), pp. 463-486.

[34] F. Radjai and S. Roux, Phys. Rev. E 51, 6177 (1995).

[35] A. Hurst, J. Cartwright, M. Huuse, R. Jonk, A. Schwab, D. Duranti, and B. Cronin, Geofluids 3, 263 (2003).

[36] H. A. Janssen, Z. Vereines Deutsch. Ingenieure 39, 1045 (1895).

[37] W. H. Press, S. A. Teukolsky, W. T. Vetterling, and B. P. Flannery, Numerical Recipes in $\mathrm{C}++$ (Cambridge University Press, Cambridge, U.K., 2002), Chap. 12, p. 505. 